\title{
ASYMPTOTIC SOLUTIONS OF LINEAR VOLTERRA INTEGRAL EQUATIONS WITH SINGULAR KERNELS
}

\author{
BY \\ J. S. W. WONG AND R. WONG(1)
}

ABSTRACT. Volterra integral equations of the form $u^{\prime}(t)=-\int_{0}^{t} a(t-\tau) u(\tau) d \tau, u(0)$ $=1$, are considered, where $a(t) \in C(0, \infty) \cap L_{1}(0,1)$. Explicit asymptotic forms are obtained for the solutions, when the kernels $a(t)$ have a specific asymptotic representation.

1. Introduction. In this paper, we study the asymptotic behavior of the solution of the linear Volterra integral equation

$$
u^{\prime}(t)=-\int_{0}^{t} a(t-\tau) u(\tau) d \tau, \quad u(0)=1,
$$

where $a(t) \in C(0, \infty) \cap L_{1}(0,1)$. By standard results on Volterra equations, we know that equation (1.1) has a unique solution in $C^{1}[0, \infty)$. We are here concerned with the problem of asymptotic stability of the zero solution of (1.1), i.e. when the solution $u(t)$ of (1.1) satisfies

$$
\lim _{t \rightarrow \infty} u(t)=0 .
$$

Equation (1.1) is a special case of a more general nonlinear equation studied by Levin [7] and Levin and Nohel [8]; they obtain sufficient conditions so that (1.2) will hold. For the linear equation (1.1), Hannsgen [5] has employed Laplace transform methods to improve Levin's result for this special case. Specifically, it is shown in [5] that if $a(t)$ satisfies

$$
(-1)^{k} a^{(k)}(t) \geq 0, \quad k=0,1,2,
$$

and $a(t) \not a\left(0_{+}\right)$, then the solution $u(t)$ of (1.1) satisfies (1.2). In this paper, we are not concerned with sufficient conditions for the asymptotic stability of (1.1) but rather with the rate of decay of solutions of (1.1) when it is known that the equation is asymptotically stable. Thus, when the kernel $a(t)$ is known to have a specific asymptotic representation, one seeks for asymptotic representation of the solution of (1.1). This approach has been investigated in a recent paper of

Received by the editors June 26, 1972.

AMS (MOS) subject classifications (1970). Primary 45A05, 45D05, 45E99; Secondary 45M05, 45M10, 33A70.

Key words and phrases. Volterra integral equation, asymptotic form, Laplace transform, completely monotonic.

(1) The first author was supported in part by Army Research Office, Durham through contract DA-ARO-D-31-124-72-G95. The second author was supported in part by National Research Council of Canada under contract A7359. 
Hannsgen [6]. A typical result is the following: suppose that $a(t)=C t^{\alpha}+b(t)$, $t \rightarrow \infty$, where $C>0, b(t)$ is completely monotonic (i.e. $(-1)^{k} b^{(k)}(t) \geq 0, k=0$, $1,2, \ldots)$ and $b(t)=o\left(t^{\delta}\right),-1<\delta<\alpha<0$; then the solution $u(t)$ admits the following asymptotic representation:

$$
u(t)=(\Gamma(\alpha+1) / C \Gamma(-\alpha-1)) t^{-\alpha-2}+O\left(t^{\varepsilon}\right), \quad t \rightarrow \infty,
$$

where $\varepsilon>-2 \alpha+\delta-2$. The method of his study is based upon Bernstein's representation for completely monotonic functions $[11$, p. 161] and indirect abelian theorems. Here we shall adopt a different approach whereby we assume that $a(t)$ possesses certain asymptotic behavior on a sector containing the positive reals $R^{+}$and obtain asymptotic representation of solutions of (1.1). This approach does not require that $a(t)$ is completely monotonic as a function of the real variable $t$ but demands more information on $a(t)$ as a function of the complex variable $t$ in a sector containing $R^{+}$. In contrast, our method of study is based upon tauberian theorems, inversion formulae for Laplace transforms and asymptotic evaluation of contour integrals. It should be pointed out that some more or less standard tauberian theorems have been used to obtain asymptotic expansions of solutions of specific Volterra equations of the form (1.1) as $t \rightarrow \infty$; see, for example, Levin and Nohel [9] and Bronikowski [1]. However, none of these results are applicable to the situations to be considered in the present paper. This is due to the fact that the Laplace transform of the solution of (1.1) often behaves like the logarithmic function near the origin. In the next section we shall prove a tauberian theorem which is quite different from the existing ones and hence is of independent interest.

2. Preliminary considerations. Let $T_{\alpha}$ denote the subset of the complex $t$-plane given by

$$
T_{\alpha}=\{t:|\arg t| \leq \alpha, t \neq 0\}, \quad 0<\alpha<\pi / 2 .
$$

Let $A(s)$ denote the Laplace transform of the function $a(t)$ given by

$$
A(s)=\int_{0}^{\infty} e^{-s t} a(t) d t
$$

We shall assume throughout this paper that the kernel function $a(t)$ satisfies the following conditions:

$\left(\mathrm{A}_{1}\right) a(t)$ is defined and analytic in $T_{\alpha}$;

$\left(\mathrm{A}_{2}\right) a(t)=O\left(t^{\eta}\right)$ as $|t| \rightarrow 0$ in $T_{\alpha}$ for some $\eta>-1$, and $a(t)=O(1)$ as $|t| \rightarrow \infty$ in $T_{\alpha}$;

$\left(\mathrm{A}_{3}\right) A(s)$ is analytic and $\operatorname{Re} A(s)$ positive on the right half-plane $\operatorname{Re} s \geq 0$, with the exception of a possible singularity at $s=0$.

Assumption $\left(A_{1}\right)$ is markedly the major difference in our approach. Here we assume the kernel function $a(t)$ defined as a function of a complex variable over 
a sector containing the positive real axis. The first part of assumption $\left(\mathbf{A}_{2}\right)$ implies that $a(t) \in L_{1}(0,1)$. These two assumptions are stronger than that imposed by Hannsgen [6]. The second part of assumption $\left(A_{2}\right)$ and assumption $\left(A_{3}\right)$, however, are weaker than those imposed in [6]. In fact, if assumption (1.3) holds then these two assumptions are clearly satisfied (see e.g. [10]). It should perhaps be pointed out that assumption $\left(A_{3}\right)$ alone is sufficient for the asymptotic stability of (1.1). On the other hand, if $a(t)$ is completely monotonic then it has an analytic continuation from $R^{+}$to the whole right half-plane.

By rotating the ray of integration in (2.2), $A(s)$ can be analytically continued in view of $\left(A_{1}\right),\left(A_{2}\right)$ from the right half-plane $\operatorname{Re} s \geq 0$ to the larger sector

$$
S_{\alpha}^{0}=\{s:|\arg s|<\pi / 2+\alpha, s \neq 0\}, \quad \alpha>0 .
$$

(See $\$ 2$, Chapter VI and $\$ 5$, Chapter VII of [4].) In the half-plane $H_{\theta}$ defined by

$$
H_{\theta}=\left\{s: \operatorname{Re}\left(s e^{i \theta}\right)>0,|\theta| \leq \alpha\right\},
$$

the function $A(s)$ is represented by the integral

$$
A(s)=\int_{0}^{\infty e^{i \theta}} a(t) e^{-s t} d t, \quad s \in H_{\theta} .
$$

By the principle of analytic continuation, the function $A(s)$ does not depend on the ray chosen for the integration. In what follows it is therefore understood that $A(s)$ is an analytic function in the sector $S_{\alpha}^{0}$ and is represented by $(2.5)$ in the halfplane $H_{\theta}$ for every $|\theta| \leq \alpha$.

Returning to (1.1) and taking Laplace transforms, we obtain

$$
\cup(s)=(s+A(s))^{-1}, \quad \operatorname{Re} s>0,
$$

where $U(s)$ denotes the Laplace transform of the solution $u(t)$. Since $A(s)$ is analytic in $S_{\alpha}^{0}, \cup(s)$ is analytic in $S_{\alpha}^{0}$ except at the zeros of $s+A(s)$. In fact, we shall now show that there exists a $0<\delta<\alpha$ such that $\cup(s)$ is analytic in

$$
S_{\delta}=\{s:|\arg s| \leq \pi / 2+\delta, s \neq 0\} .
$$

From assumption $\left(\mathrm{A}_{3}\right)$ it follows that $s+A(s)$ has no zero in the right half-plane $\{s: \operatorname{Re} s \geq 0\}$. (Note that $A(s)$ may have a singularity at $s=0$.) By $\left(\mathrm{A}_{2}\right)$, we know from the representation (2.5) that

$$
A(s)=O\left(s^{-\varepsilon}\right), \quad s \rightarrow \infty \text { in } H_{\theta},
$$

where $\varepsilon=\min (1+\eta, 1)$. The estimate (2.8) shows that $s+A(s)$ cannot have zeros in $S_{\alpha}^{0}$ for large absolute values of $s$. Since an analytic function (not identically zero) can have only a finite number of zeros in any compact subset of the plane, there must exist $\delta>0$ such that $s+A(s)$ has no zeros in $S_{\delta}$. 
The complex inversion formula for Laplace transforms gives

$$
u(t)=\frac{1}{2 \pi i} \int_{c-i \infty}^{c+i \infty} \cup(s) e^{s t} d s, \quad t>0,
$$

for some $c>0$, the integral above is taken as a principal value. (For a justification of (2.9), see [5].) Since $U(s)$ is analytic in $S_{s}$ and $U(s)=O\left(s^{-1}\right)$ as $s \rightarrow \infty$ in $S_{\delta}$, the Cauchy's theorem then yields

$$
u(t)=\frac{1}{2 \pi i} \int_{C} \cup(s) e^{s t} d s, \quad t>0,
$$

where the contour $C$ (Figure 1) is described as follows: for any $r>0, C$ consists of the radial lines $\theta=(\pi / 2+\delta),|s|>r, 0<\delta<\alpha$ and the portion of the semicircle of radius $r$ shown. The integral in (2.10) is again taken as a principal value.

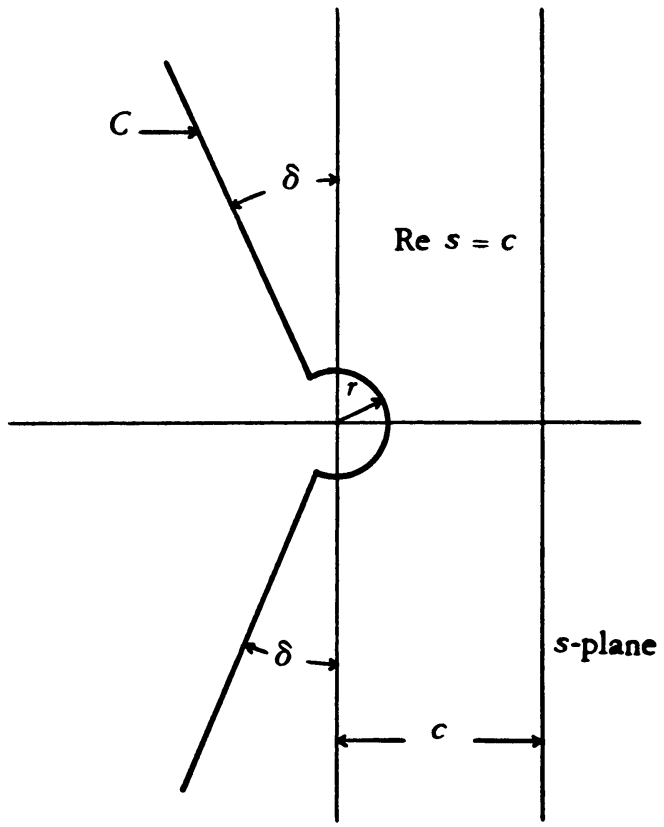

Figure 1. Contour $C$

The principal tool for our subsequent analysis is the following Tauberian result.

Theorem 1. Assume that

(i) $F(s)$ is analytic in $S_{\delta}$,

(ii) $F(s) \rightarrow 0$ uniformly as $s \rightarrow \infty$ in $S_{\delta}$,

(iii) the inverse transform of $F(s)$

$$
f(t)=\frac{1}{2 \pi i} \int_{c-i \infty}^{c+i \infty} F(s) e^{s t} d s
$$


exists for $t>0$, at least as a principal value for some $c>0$,

(iv) $F(s)$ has the following limiting behavior

$$
F(s)=s^{\lambda-1}(\log s)^{\alpha}\left\{C_{0}+O\left(s^{e}\right)\right\}, \quad \varepsilon>0,
$$

as $s \rightarrow 0$ in $S_{\delta}$ where $\alpha \neq 0$ and $\lambda \neq 1,2, \ldots$.

Then the function $f(t)$ given in (2.11) has the following asymptotic formula

(2.13) $f(t)=\frac{(-\log t)^{\alpha}}{t^{\lambda}}\left\{\frac{C_{0}}{\Gamma(1-\lambda)}-D\left(\Gamma^{-1}(1-\lambda)\right) \frac{\alpha C_{0}}{\log t}+O\left(\frac{1}{\log ^{2} t}\right)\right\}$

as $t \rightarrow+\infty$, where $D=d / d \lambda$.

Proof. Assumption (ii) implies by the Cauchy theorem that

$$
f(t)=\frac{1}{2 \pi i} \int_{C} F(s) e^{s t} d s,
$$

where $C$ is the contour shown in Figure 1. For fixed $r>0$, the semicircle $|s|=r$, $|\arg s| \leq \pi / 2+\delta$ and the two radial lines arg $s= \pm(\pi / 2+\delta),|s|>r$ form such a contour $C$. We refer to the first part of this contour as $A$ and the second part as $B$.

On $B, F(s)$ is bounded, say by $M$, therefore we have

$$
\begin{aligned}
\left|\frac{1}{2 \pi i} \int_{B} F(s) e^{s t} d s\right| & \leq \frac{M}{\pi} \int_{r}^{\infty} \exp (u t \cos (\pi / 2+\delta)) d u \\
& =\frac{M}{\pi} \int_{r}^{\infty} e^{-u t \sin \delta} d u=\frac{M}{\pi} \frac{e^{-t r \sin \delta}}{t \sin \delta}=o\left(e^{-e t}\right),
\end{aligned}
$$

as $t \rightarrow+\infty$, where $\varepsilon=r \sin \delta>0$. Using (2.12), we can estimate the integral on contour $A$ as follows

$$
\frac{1}{2 \pi i} \int_{A} F(s) e^{s t} d s=\frac{C_{0}}{2 \pi i} \int_{A} s^{\lambda-1}(\log s)^{\alpha} e^{s t} d s+R(t)
$$

where $R(t)$ satisfies the inequality

$$
|R(t)| \leq \frac{1}{2 \pi} \int_{A}\left|s^{\lambda+e-1}(\log s)^{\alpha} e^{s t} d s\right| .
$$

A simple estimate yields

$$
R(t)=O\left((\log t)^{\alpha} / t^{\lambda+\varepsilon}\right), \quad \text { as } t \rightarrow \infty .
$$

Combining estimates (2.15) and (2.18), we obtain the following,

$$
f(t)=\frac{C_{0}}{2 \pi i} \int_{A} s^{\lambda-1}(\log s)^{\alpha} e^{s t} d s+O\left(\frac{(\log t)^{\alpha}}{t^{\lambda+e}}\right), \quad \text { as } t \rightarrow \infty
$$


The integral in (2.19) has been studied by Wyman and Wong [13] and has the asymptotic representation

$$
\begin{aligned}
\frac{1}{2 \pi i} \int_{A} s^{\lambda-1}(\log s)^{\alpha} e^{s t} d s \\
=\frac{(-\log t)^{\alpha}}{t^{\lambda}}\left[\frac{1}{\Gamma(1-\lambda)}+D\left(\Gamma^{-1}(1-\lambda)\right) \frac{\alpha}{\log t}+O\left(\frac{1}{(\log t)^{2}}\right)\right]
\end{aligned}
$$

as $t \rightarrow \infty$. Thus, (2.19) and (2.20) together give the desired result (2.13).

We note that the asymptotic representation given by $(2.13)$ is independent of $\lambda$. Therefore, we may let $\lambda \rightarrow m, m=1,2,3, \ldots$, and deduce the following

Corollary 1. Under the hypothesis of Theorem 1, we have

$$
f(t)=\left(C_{0} / t^{\lambda}\right)(-\log t)^{\alpha-1}\left[(-1)^{\lambda} \Gamma(\lambda) \alpha+O(1 / \log t)\right]
$$

for $\lambda=1,2,3, \ldots$.

As an immediate application of our corollary, we obtain the following result concerning solutions of equation (1.1).

Theorem 2. Let $a(t)=(t+1)^{-1}$ in equation (1.1). Then the solution $u(t)$ of (1.1) has the asymptotic representation

$$
u(t)=\left(t \log ^{2}(t / d)\right)^{-1}+O\left\{t^{-1}\left(\log ^{-3}(t / d)\right)\right\},
$$

as $t \rightarrow \infty$, where $d=e^{\gamma}$ and $\gamma$ is the Euler constant. by

$$
A(s)=\int_{0}^{\infty} \frac{e^{-s t}}{1+t} d t=\Psi(1,1, s),
$$

$\operatorname{Re} s>0$, where $\Psi$ is the confluent hypogeometric function $[3, \S 6.5]$. It is also known that the function $\Psi(1,1, s)$ is analytic in the complex s-plane cut along the negative real axis and has the following behavior near $s=0$ :

$$
A(s)=\Psi(1,1, s)=-\log s-\gamma+O\left(s(\log s)^{-1}\right),
$$

$s \rightarrow 0$ (see $[3, \S 6.8]$ and note that the constant $\gamma$ is missing there). Thus, from (2.6) we have

$$
\cup(s)=-(\log s d)^{-1}(1+O(s))
$$

as $s \rightarrow 0$ in the sector $|\arg s| \leq \pi-\Delta<\pi$. Applying Corollary 1 to (2.25), we obtain the desired result $(2.22)$. 
We note that the asymptotic representation (2.22) concerning solution $u(t)$ of (1.1) is an improvement over an earlier result reported by Hannsgen [6] who proved

$$
u(t)=\left(t \log ^{2}(t / d)\right)^{-1}+O\left\{t^{-1}(\log (t / d))^{-3+\varepsilon}\right\},
$$

where $0<\varepsilon<1$ and the constant $d$ is also not specified.

3. The case when $a(t) \sim t^{\gamma}, \gamma<0$. We present here in detail our analysis for the case when $a(t)$ satisfies the asymptotic form

$$
a(t) \sim t^{\gamma}, \quad t \rightarrow \infty \text { in } T_{\alpha},
$$

where $\gamma<0$. The results are given by the following four theorems treating (i) $-1<\gamma<0$, (ii) $\gamma=-1$, (iii) $\gamma<-1$ and $\gamma \neq-2,-3, \ldots$, and (iv) $\gamma=-2$, $-3, \ldots$ respectively.

Theorem 3. Let

$$
a(t)=\frac{C}{\Gamma(\gamma+1)} t^{\gamma}+b(t), \quad t \in T_{\alpha},
$$

where $C>0, b(t)=O\left(t^{\rho}\right)$ as $t \rightarrow \infty$ in $T_{a}$ and $-1<\rho<\gamma<0$. Then the solution $u(t)$ of $(1.1)$ satisfies

$$
u(t)=t^{-2-\gamma} / C \Gamma(-1-\gamma)+O\left(t^{e}\right), \quad t \rightarrow \infty,
$$

where $\varepsilon=-2-2 \gamma+\rho$.

Proof. Taking the Laplace transforms of (3.2) we have

$$
A(s)=C s^{-\gamma-1}+\int_{0}^{\infty} b(t) e^{-s t} d t,
$$

$\operatorname{Re} s>0$. Denote by $B(s)$ the Laplace transform of $b(t)$. Since $b(t) \in L_{1}(0,1)$ and $b(t)=O\left(t^{p}\right)$ as $t \rightarrow \infty$, it is easy to see that

$$
B(s)=O\left(s^{-\rho-1}\right), \text { as } s \rightarrow 0,
$$

in $|\arg s| \leq \pi / 2-\Delta<\pi / 2$. Hence, it follows from (3.4) and (3.5) that

$$
A(s)=C s^{-\gamma-1}\left(1+O\left(s^{\gamma-\rho}\right)\right) \text {, }
$$

as $s \rightarrow 0$ in $|\arg s| \leq \pi / 2-\Delta$. Substituting (3.6) into (2.6), we find

$$
\cup(s)=C^{-1} s^{\gamma+1}\left[1+O\left(s^{\gamma-p}\right)\right],
$$

as $s \rightarrow 0$ in $|\arg s| \leq \pi / 2-\Delta$.

In view of the analyticity of $a(t)$ in $T_{\alpha}$, we can write $A(s)$ and $B(s)$ in terms of a contour integral on a ray with $\arg t=\theta$ where $\theta \in[-\alpha, \alpha]$. Such a representa- 
tion, as given by (2.5), is valid for $s \in H_{\theta}$. Following a similar argument as that given above, we conclude that the formula (3.7) for $U(s)$ is also valid in $\left|\arg \left(s e^{i \theta}\right)\right| \leq \pi / 2-\Delta$. Since $\theta$ is an arbitrary number in $[-\alpha, \alpha],(3.7)$ holds for $s \in S_{\delta}, 0<\delta<\alpha$. Substituting (3.7) into (2.10), the desired result (3.3) now follows by a simple complex integration.

Remark 3.1. Hannsgen [6] obtained the conclusion (3.3) by an entirely different technique. His condition on $\varepsilon>-2-2 \gamma+\rho$ is more restrictive.

Theorem 4. Let

$$
a(t)=C t^{-1}+b(t), \quad t \in T_{\alpha},
$$

where $b(t)=O\left(t^{-\rho}\right)$ as $t \rightarrow \infty$ in $T_{\alpha}$ and $\rho>1$. Then the solution $u(t)$ of (1.1) satisfies

$$
u(t)=\frac{1}{C t \log ^{2} t}+O\left(\frac{1}{t \log ^{3} t}\right), \quad \text { as } t \rightarrow \infty
$$

Proof. Since $t^{-1} \notin L_{1}(0,1)$, we must consider the Laplace transform of $a(t)$ in two parts, namely,

$$
A(s)=\int_{0}^{1} a(t) e^{-s t} d t+\int_{1}^{\infty} a(t) e^{-s t} d t
$$

As $a(t) \in L_{1}(0,1)$, the first integral in (3.10) is an entire function of $s$ which we denote by $\varphi(s)$. The second integral in (3.10) can be estimated by using the limiting behavior of $a(t)$ given in (3.8). Observe first that for $\operatorname{Re} s>0$,

$$
\int_{1}^{\infty} t^{-1} e^{-s t} d t=e^{-s} \Psi(1,1, s)
$$

The above integral has an analytic continuation into the angle $\mid$ arg $s \mid \leq \pi-\Delta$ and in the neighborhood of $s=0$ it has the same asymptotic behavior as $\Psi(1,1, s)$, as can be seen by a change of variable and comparison with (2.24).

By hypothesis, $b(t)=O\left(t^{-\rho}\right)$ as $t \rightarrow \infty$ and $\rho>1$. Hence

$$
\int_{1}^{\infty} b(t) e^{-s t} d t=O(1)
$$

as $s \rightarrow 0$ in $|\arg s| \leq \pi / 2-\Delta$. Combining (3.11) and (3.12) in (3.10), we obtain

$$
s+A(s)=C \log (1 / s)+\text { Constant }+O\left(s^{e}\right)
$$

as $s \rightarrow 0$ in $|\arg s| \leq \pi / 2-\Delta$, where $0<\varepsilon<1$.

By an argument similar to that used in the proof of Theorem 3 we argue along any ray arg $t=\theta, \theta \in[-\alpha, \alpha]$ instead of the nonnegative real axis so that (3.13) is valid for $\left|\arg \left(s e^{i \theta}\right)\right| \leq \pi / 2-\Delta$. By varying $\theta$, it follows that (3.13) holds for $s \in S_{\delta}$. A simple calculation then yields 


$$
U(s)=-\frac{1}{C \log s}\left\{1+\frac{\text { Constant }}{\log s}+O\left(\frac{1}{\log ^{2} s}\right)\right\},
$$

as $s \rightarrow 0$ in $S_{8}$. For each term above in (3.14), we appeal to our main Corollary 1 , and obtain

$$
u(t)=\frac{1}{C t \log ^{2} t}+O\left(\frac{1}{t \log ^{3} t}\right)+\frac{\text { Constant }}{t \log ^{3} t}+O\left(\frac{1}{t \log ^{4} t}\right)+R(t),
$$

where

$$
|R(t)| \leq \frac{K}{2 \pi} \int_{C}\left|\log ^{-3} s e^{s t} d s\right|=O\left(\frac{1}{t \log ^{3} t}\right) .
$$

Grouping the appropriate $\boldsymbol{O}$-terms above, we obtain the desired conclusion (3.9).

Remark 3.2. It should perhaps be noted that Theorem 2 can be considered as a special case of Theorem 4 .

Theorem 5. Let

$$
a(t)=C t^{\gamma}+b(t), \quad t \in T_{\alpha},
$$

where $\gamma<-1$ and $\gamma \neq-2,-3, \ldots$ and $b(t)=O\left(t^{\gamma-1}\right)$ as $t \rightarrow \infty$ in $T_{\alpha}$. Then the solution $u(t)$ of $(1.1)$ satisfies

$$
u(t)=-C t^{\gamma} / a_{0}^{2}+O\left(1 / t^{N+1}\right),
$$

where $-(N+1)<\gamma<-N$ and $a_{0}=\int_{0}^{\infty} a(t) d t$.

Proof. For fixed $\gamma$, there exists $N>0$ such that $-(N+1)<\gamma<-N$. Using integration by parts, we find

$$
\begin{aligned}
\int_{1}^{\infty} t^{\gamma} e^{-s t} d t & =\frac{s^{N}}{(\gamma+1) \cdots(\gamma+N)} \int_{1}^{\infty} t^{\gamma+N} e^{-s t} d t+\varphi_{1}(s) \\
& =\frac{s^{N} \Gamma(\gamma+N+1)}{(\gamma+1) \cdots(\gamma+N) s^{\gamma+N+1}}-\int_{0}^{1} t^{\gamma+N} e^{-s t} d t+\varphi_{1}(s) \\
& =\frac{\Gamma(\gamma+1)}{s^{\gamma+1}}+\varphi_{2}(s) \quad(\operatorname{Re} s>0),
\end{aligned}
$$

where $\varphi_{1}(s), \varphi_{2}(s)$ are appropriate entire functions. Thus, we may write $A(s)$ in the following form:

$$
A(s)=\frac{C \Gamma(\gamma+1)}{s^{\gamma+1}}+\int_{1}^{\infty} b(t) e^{-s t} d t+\varphi_{3}(s), \quad \operatorname{Re} s>0,
$$

where $\varphi_{3}(s)$ is again some entire function. Since $b(t)=O\left(t^{r-1}\right)$ as $t \rightarrow \infty$, we can easily prove the following relation

$$
\int_{1}^{\infty} b(t) e^{-s t} d t=\sum_{n=1}^{N-1} \frac{(-s)^{n}}{n !} \mu_{n}+O\left(s^{N}\right), \quad s \rightarrow 0,
$$


where $\mu_{n}$ are constants depending only on $b(t)$. Note that (3.18) is valid for $|\arg s| \leq \pi / 2-\Delta, \Delta>0$. Combining (3.17) and (3.18) we find

$$
s+A(s)=\sum_{n=0}^{N-1} a_{n} s^{n}+C \Gamma(\gamma+1) s^{-\gamma-1}+O\left(s^{N}\right)
$$

as $s \rightarrow 0$ in $|\arg s| \leq \pi / 2-\Delta, \Delta>0$, where $a_{n}, n=0,1, \ldots, N-1$, are again constants. Letting $s \rightarrow 0$ in (3.19), we find $a_{0}=\int_{0}^{\infty} a(t) d t$. From (3.19) and (2.6), we obtain

$$
U(s)=-\frac{C}{a_{0}^{2}} \frac{\Gamma(\gamma+1)}{s^{\gamma+1}}+\sum_{n=0}^{N-1} b_{n} s^{n}+O\left(s^{N}\right), \quad \operatorname{Re} s>0,
$$

as $s \rightarrow 0$. The validity of (3.20) can be extended in the usual way to $S_{\delta}$ for some $\delta>0$. Finally a simple inversion using formula (2.10) yields the desired result (3.16).

Theorem 6. Let

$$
a(t)=C t^{-n}+b(t), \quad t \in T_{\alpha},
$$

where $n=2,3, \ldots$ and $b(t)=O\left(t^{-\eta}\right)$ as $t \rightarrow \infty$ in $T_{\alpha}, \eta>n+1$. Then the solution $u(t)$ of $(1.1)$ satisfies

$$
u(t)=-C t^{-n} / a_{0}^{2}+O\left(1 / t^{n} \log t\right), \quad t \rightarrow \infty,
$$

where $a_{0}=\int_{0}^{\infty} a(t) d t$.

Proof. The argument parallels the proof of Theorem 5. First, we note that

$$
\int_{1}^{\infty} t^{-n} e^{-s t} d t=e^{-s}\left\{\frac{1}{n-1}-\cdots+\frac{(-1)^{n-2} s^{n-2}}{(n-1) !}\right\}+\frac{(-s)^{n-1}}{(n-1) !} \int_{1}^{\infty} t^{-1} e^{-s t} d t
$$

Thus, on account of (3.11) and (2.24), we have from (3.21)

$$
A(s)=\varphi_{4}(s)+C \frac{(-s)^{n-1}}{(n-1) !} \log \frac{1}{s}+\int_{1}^{\infty} b(t) e^{-s t} d t, \quad \text { Re } s>0 .
$$

Estimate (3.18) is also valid here for the last term in (3.23). Hence, we deduce from (3.23) the following

$$
s+A(s)=\sum_{k=0}^{n-1} a_{k} s^{k}+C \frac{(-s)^{n-1}}{(n-1) !} \log \frac{1}{s}+O\left(s^{n}\right) .
$$

The validity of (3.24) can be extended in the same way as before to $S_{\delta}$. From (2.6) and (3.24) above, we obtain

$$
U(s)=\frac{1}{a_{0}}\left\{\sum_{k=0}^{n-1} b_{k} s^{k}-\frac{C(-s)^{n-1}}{a_{0}(n-1) !} \log \frac{1}{s}+O\left(s^{n}\right)\right\},
$$


from which the desired result (3.22) follows readily from Corollary 1.

4. Kernels involving logarithms. In this section, we shall consider an extension of Theorems 3 and 4 to those kernels $a(t)$ which involve logarithms in the following form:

$$
a(t) \sim C t^{\gamma}(\log t)^{0}, \quad t \rightarrow \infty \text { in } T_{\alpha},
$$

where $-1 \leq \gamma<0$ and $\sigma$ is some real number. Before proceeding to these results, we need the following lemma.

Lemma. Let $\arg c=\theta$ and $|c|>1$. Then for any $\gamma>-1$ and $\sigma$ real

$$
\int_{c}^{\infty e^{10}} t^{\gamma}(\log t)^{\sigma} e^{-s t} d t=\frac{(-\log s)^{\sigma}}{s^{\gamma+1}}\left\{\Gamma(\gamma+1)+O\left(\frac{1}{\log s}\right)\right\}
$$

as $s \rightarrow 0$ in $H_{\theta}$.

This result is given in [12] for the case $\theta=0$, but a slight modification of the given proof will yield the desired extension.

Theorem 7. Let $-1<\gamma<0$ and $\sigma$ real but arbitrary. Assume that $a(t)$ satisfies

$$
a(t)=(C / \Gamma(\gamma+1)) t^{\gamma}(\log t)^{\sigma}+b(t), \quad t \in T_{\alpha},
$$

where $b(t)=O\left(t^{\gamma}(\log t)^{\sigma-\delta}\right)$ as $t \rightarrow \infty$ in $T_{\alpha}$ and $\delta>0$. Then the solution $u(t)$ of (2.1) satisfies

$$
u(t)=\frac{t^{-\gamma-2}}{C \Gamma(-\gamma-1)(\log t)^{\sigma}}\left\{1+O\left(\frac{1}{(\log t)^{e}}\right)\right\}
$$

as $t \rightarrow \infty$ for some positive number $\varepsilon$.

Proof. Let $c>1$ and from (4.3) write $A(s)$ in the following form

$$
\begin{aligned}
A(s)= & \int_{0}^{c} a(t) e^{-s t} d t+\frac{C}{\Gamma(\gamma+1)} \int_{c}^{\infty} t^{\gamma}(\log t)^{0} e^{-s t} d t \\
& +\int_{c}^{\infty} b(t) e^{-s t} d t
\end{aligned}
$$

The last integral can be estimated as

$$
\int_{c}^{\infty} b(t) e^{-s t} d t=O\left(s^{-\gamma-1}(\log s)^{0-\delta}\right),
$$

as $s \rightarrow 0$ in $H_{0}$. By the above lemma, we have

$$
s+A(s)=\frac{C(-\log s)^{o}}{s^{\gamma+1}}\left\{1+O\left(\frac{1}{(\log s)^{z^{\prime}}}\right)\right\}
$$


for some $\varepsilon^{\prime}>0$, and hence

$$
U(s)=\frac{s^{\gamma+1}}{C(-\log s)^{\sigma}}\left\{1+o\left(\frac{1}{(\log s)^{e^{\prime}}}\right)\right\},
$$

as $s \rightarrow 0$ in $H_{0}$. The validity of (4.8) can be extended in the usual way to $S_{8}$. An application of Theorem 1 to (4.8) then yields the desired result (4.4).

Theorem 8. Let $\sigma>-1$ and

$$
a(t)=C t^{-1}(\log t)^{\sigma}+b(t), \quad t \in T_{\alpha},
$$

where $b(t)=O\left(t^{-1}(\log t)^{\sigma-\eta}\right)$ and $\eta>0$. Then the solution $u(t)$ of $(2.1)$ satisfies

$$
u(t)=\frac{(\sigma+1)^{2}}{C t(\log t)^{\sigma+2}}\left\{1+o\left(\frac{1}{(\log t)^{e}}\right)\right\}
$$

as $t \rightarrow \infty$, for some $\varepsilon>0$.

Proof. As integration by parts gives

$$
\int_{c}^{\infty} t^{-1}(\log t)^{\sigma} e^{-s t} d t=\varphi(s)+\frac{s}{\sigma+1} \int_{c}^{\infty}(\log t)^{\sigma+1} e^{-s t} d t,
$$

where $\varphi(s)$ is an appropriate entire function. To the last integral in (4.11) we can apply the result of our lemma. Thus

$$
\int_{c}^{\infty} t^{-1}(\log t)^{0} e^{-s t} d t=\varphi(s)+\frac{(-\log s)^{\sigma+1}}{\sigma+1}+O\left((\log s)^{0}\right),
$$

as $s \rightarrow 0$ in $H_{0}$. Similarly, we obtain

$$
\int_{c}^{\infty} b(t) e^{-s t} d t=O\left((-\log s)^{\sigma-\eta+1}\right),
$$

as $s \rightarrow 0$ in $H_{0}$. Using (4.9), (4.12) and (4.13), one easily obtains

$$
s+A(s)=\frac{C}{\sigma+1}(-\log s)^{\sigma+1}\left\{1+O\left(\frac{1}{(\log s)^{e}}\right)\right\}
$$

as $s \rightarrow 0$ in $H_{0}$ where $\varepsilon$ is some positive number. Extending the range of validity in the usual way, we have from (4.14)

$$
U(s)=\frac{(\sigma+1)}{C}(-\log s)^{-\sigma-1}\left\{1+o\left(\frac{1}{(\log s)^{2}}\right)\right\}
$$

as $s \rightarrow 0$ in $S_{8}$. An application of Corollary 1 to (4.15) readily yields the desired result (4.10).

Theorem 9. Let $\sigma<-1$ and assume that

$$
a(t)=C t^{-1}(\log t)^{0}+b(t), \quad t \in T_{a},
$$


where $b(t)=O\left(t^{-1}(\log t)^{\sigma-\eta}\right)$ and $\eta>0$. Then, the solution $u(t)$ of (2.1) satisfies

$$
u(t)=-\frac{C}{a_{0}^{2}} \frac{(\log t)^{o}}{t}\left\{1+O\left(\frac{1}{(\log t)^{2}}\right)\right\}
$$

where $\varepsilon>0$ and $a_{0}=\int_{0}^{\infty} a(t) d t$.

Proof. Let $\arg c=\theta$ and $|c|>1$. Write

$$
A(s)=\int_{0}^{c} a(t) e^{-s t} d t+C \int_{c}^{\infty e^{i 0}} t^{-1}(\log t)^{0} e^{-s t} d t+\int_{c}^{\infty e^{\prime 0}} b(t) e^{-s t} d t
$$

It is clear that (4.12) and (4.13) remain valid if the integration is performed on a ray $\arg t=\theta$ rather than on the real axis. Hence, (4.18) together with (4.12) and (4.13) yield

$$
A(s)=\varphi(s)+\frac{C}{\sigma+1}(-\log s)^{\sigma+1}\left\{1+o\left(\frac{1}{(\log s)^{e}}\right)\right\}
$$

as $s \rightarrow 0$ in $S_{8}$ for some $\varepsilon>0$. Since $\sigma+1<0$, so by letting $s \rightarrow 0$ in (4.19) we have $\varphi(0)=a_{0}$. Therefore, we find

$$
\cup(s)=(s+A(s))^{-1}=\frac{1}{a_{0}}-\frac{C}{a_{0}^{2}(\sigma+1)}(-\log s)^{\sigma+1}+O\left((-\log s)^{\sigma+1-\varepsilon^{\prime}}\right)
$$

for some $\varepsilon^{\prime}>0$, as $s \rightarrow 0$ in $S_{8}$. By Corollary 1 again, we readily have the desired result (4.17).

5. Examples. In this final section, we shall present some specific asymptotic solutions to equation (2.1) when the kernel $a(t)$ is explicitly given. These examples serve to verify the general results presented in $\$ \S 3$ and 4 and, at the same time, mark the boundary of our results.

Example 1. Consider $a(t)=(t(t+2))^{-1 / 2}$. It is well known that

$$
A(s)=e^{s} K_{0}(s),
$$

where $K_{0}(s)$ is the modified Bessel function of the third kind,

$$
K_{0}(s)=\sum_{k=0}^{\infty} \frac{1}{(k !)^{2}}\left(\frac{s}{2}\right)^{2 k}\left[\Psi(k+1)-\log \frac{s}{2}\right]
$$

where $\Psi(\lambda)=\Gamma^{\prime}(\lambda) / \Gamma(\lambda)$. Since $\Psi(1)=-\gamma$ (see [3, \$1.7]), (5.2) implies

$$
K_{0}(s)=\log (1 / s)+(\log 2-\gamma)+O\left(s^{2} \log s\right),
$$

as $s \rightarrow 0$. Therefore, (5.1) and (5.3) imply

$$
s+A(s)=\log (1 / s)+(\log 2-\gamma)+s \log (1 / s)+O(s),
$$


from which it follows that

$$
\cup(s)=-\frac{1}{\log s}\left\{1+\frac{\log 2-\gamma}{\log s}+O\left(\frac{1}{\log ^{2} s}\right)\right\}
$$

as $s \rightarrow 0$ in $S_{8}$. An application of Corollary 1 to (5.4) then yields

$$
u(t)=1 / t \log ^{2} t+O\left(1 / t \log ^{3} t\right), \quad t \rightarrow \infty .
$$

This result agrees well with Theorem 4.

Example 2. Consider $a(t)=\pi^{-1} t^{-1 / 2}(t+1)^{-1}$. The Laplace transform of $a(t)$ is known to be

$$
A(s)=e^{s} \operatorname{Erf}_{\mathrm{c}}(\sqrt{ } s)
$$

where Erf $_{c}$ is the complementary error function defined by

$$
\operatorname{Erf}_{\mathrm{c}}(s)=\frac{2}{\sqrt{ } \pi} \int_{s}^{\infty} e^{-t^{2}} d t
$$

It is well known that $\operatorname{Erf}_{c} s$ has the following series representation valid for all complex s:

$$
\operatorname{Erf}_{c}(s)=1-\frac{2}{\sqrt{ } \pi} \sum_{n=0}^{\infty} \frac{(-1)^{n} s^{2 n+1}}{n !(2 n+1)}
$$

From (5.7) and (5.6), we obtain $s+A(s)=1-2 \pi^{-1 / 2} s^{1 / 2}+O(s)$, as $s \rightarrow 0$ in $S_{\delta}$; hence

$$
\cup(s)=1+2 \pi^{-1 / 2} s^{1 / 2}+O(s),
$$

as $s \rightarrow 0$ in $S_{\delta}$, from which it follows that

$$
u(t)=-t^{-3 / 2} / \pi+O\left(1 / t^{2}\right), \quad t \rightarrow \infty .
$$

The asymptotic solution (5.8) readily agrees with Theorem 5, as a simple integration will show that $\int_{0}^{\infty} d t / \sqrt{t}(t+1)=\pi$.

Example 3. Consider $a(t)=(t+1)^{-2}$. It is well known that

$$
A(s)=\int_{0}^{\infty} \frac{e^{-s t}}{(t+1)^{2}} d t=e^{s} s \Gamma(-1, s),
$$

where $\Gamma(\alpha, s)$ is the incomplete Gamma function given by $\Gamma(\alpha, s)=\int_{s}^{\infty} u^{\alpha-1} e^{-u} d u$ which is analytic in the $s$-plane cut along the negative real axis. To find out the asymptotic representation for $A(s)$, we integrate by parts and obtain

$$
A(s)=1-e^{s} s \int_{1}^{\infty} \frac{e^{-s u}}{u} d u=1-s \Psi(1,1, s)
$$


From (5.10) and the asymptotic representation (2.24) for $\Psi(1,1, s)$, we find

$$
s+A(s)=1+s \log s+(1+\gamma) s+O\left(s^{e}\right),
$$

as $s \rightarrow 0$ in $S_{8}$, where $\delta>0$ and $\varepsilon>1$. Consequently, (5.11) yields

$$
U(s)=1-s \log s-(1+\gamma) s+O\left(s^{e^{\prime}}\right),
$$

as $s \rightarrow 0$ in $S_{\delta}$ where $\varepsilon^{\prime}>1$. From Corollary 1 again, we obtain

$$
u(t)=-1 / t^{2}+O\left(1 / t^{2} \log t\right), \quad t \rightarrow \infty .
$$

This result is also in agreement with Theorem 6 since we can easily verify that the constant $a_{0}=\int_{0}^{\infty} d t /(t+1)^{2}=1$.

Example 4. Consider the case when $a(t)$ is given by

$$
a(t)=t^{-1 / 2}\left\{\frac{\Gamma^{\prime}(1 / 2)}{\Gamma^{2}(1 / 2)}-\frac{\log t}{\Gamma(1 / 2)}\right\}
$$

It is easy to verify that its Laplace transform is

$$
A(s)=\log s / \sqrt{s}, \quad \operatorname{Re} s>0 .
$$

Although the function $a(t)$ given by (5.12) does not satisfy the convexity condition, it is easy to see from (5.13) that $s+A(s)$ has no zeros in $S_{\delta}$ for some $\delta>0$. Hence

$$
\cup(s)=\sqrt{s} /\left(s^{3 / 2}+\log s\right), \quad s \in S_{\delta}, \delta>0
$$

and in fact

$$
\cup(s)=\frac{\sqrt{s}}{\log s}\left(1+O\left(\frac{s^{3 / 2}}{\log s}\right)\right)
$$

as $s \rightarrow 0$ in $S_{\delta}$. Thus we have from Theorem 1

$$
u(t)=\frac{t^{-3 / 2}}{\sqrt{ } \pi(-\log t)}\left\{1+O\left(\frac{1}{(\log t)^{\sigma}}\right)\right\}, \quad t \rightarrow \infty,
$$

for some positive constant $\sigma$. (Note: $\Gamma(1 / 2)=\sqrt{ } \pi$ ). Estimate $(5.15)$ can be obtained also directly from Theorem 7 .

Example 5. Consider $a(t)=C+t^{-0}, 0<0<1$. The Laplace transform $A(s)=C s^{-1}+\Gamma(1-\sigma) s^{\sigma-1}$ and the Laplace transform $\cup(s)$ of the solution is given by

$$
\cup(s)=\frac{s}{s^{2}+C+\Gamma(1-\sigma) s^{0}}=\frac{s}{s^{2}+C}-\frac{\Gamma(1-\sigma) s^{0+1}}{\left(s^{2}+C\right)\left(s^{2}+C+\Gamma(1-\sigma) s^{0}\right)} .
$$


Taking inverse transform of $U(s)$, one obtains readily

$$
u(t)=\cos t+O\left(t^{0-2}\right), \quad t \rightarrow \infty .
$$

Conclusion (5.16) shows that asymptotic solution to equation (1.1) is available even when the equation itself is not asymptotically stable.

\section{REFERENCES}

1. T. A. Bronikowski, An integrodifferential system which occurs in reactor dynamics, Arch. Rational Mech. Anal. 37 (1970), 363-380. MR 42 \#3527.

2. G. Doetsch, Handbuch der Laplace-Transformation. Band II. Anwendungen der Laplace-Transformation, Birkhauser Verlag, Basel, 1955. MR 18, 35.

3. A. Erdelyi et al., Higher transcendental functions. Vol. I. The hypergeometric function, Legendre functions, McGraw-Hill, New York, 1953. MR 15, 419.

4. M. A. Evgrafov, Analytic functions, "Nauka", Moscow, 1965; English transl., Saunders, Philadelphia, Pa., 1966. MR 32 \#5843; MR 33 \#5849.

5. K. B. Hannsgen, Indirect abelian theorems and a linear Volterra equation, Trans. Amer. Math. Soc. 142 (1969), 539-555. MR 39 \# 7364.

6. , A Volterra equation with completely monotonic convolution kernel, J. Math. Anal. Appl. 31 (1970), 459-471. MR 42 \#806.

7. J. J. Levin, The asymptotic behavior of the solution of a Volterra equation, Proc. Amer. Math. Soc. 14 (1963), 534-541. MR 27 \#2824.

8. J. J. Levin and J. A. Nohel, Perturbations of a nonlinear Volterra equation, Michigan Math. J. 12 (1965), 431-447. MR 32 \#336.

9. - On a system of integrodifferential equations occurring in reactor dynamics, J. Math. Mech. 9 (1960), 347-368. MR 22 \#8301.

10. R. C. MacCamy and J. S. W. Wong, Stability theorems for some functional differential equations, Trans. Amer. Math. Soc. 164 (1972), 1-37.

11. D. V. Widder, The Laplace transform, Princeton Math. Series, vol. 6, Princeton Univ. Press, Princeton, N. J., 1941. MR 3, 232.

12. R. Wong, On a Laplace integral involving logarithms, SIAM J. Math. Anal. 1 (1970), 360-364. MR. 43 \#7842.

13. M. Wyman and R. Wong, The asymptotic behavior of $\mu(z, \beta, \alpha)$, Canad. J. Math. 21 (1969), 1013-1023. MR 39 \#5835.

Department of Mathematics, University of Iowa, Iowa City, Iowa 52240

Department of Mathematics, University of Manitoba, Winnipeg, Manttoba, Canada 